\title{
Stimulus size and acuity in information processing
}

\author{
DEREK W. SCHULTZ and CHARLES W. ERIKSEN \\ University of Illinois at Urbana-Champaign, Champaign, Illinois 61820
}

\begin{abstract}
Ten subjects served in a speeded-recognition task wherein letter targets were varied from $.143 \mathrm{deg}$ to $2.14 \mathrm{deg}$ of visual angle in height. Identification latencies were found to decrease on the order of $40 \mathrm{msec}$ for both blocked and randomized presentations of sizes as target size increased. Beyond a size of approximately $.75 \mathrm{deg}$ of visual angle, reaction time became asymptotic, and blocked presentation of target size was judged to be faster than randomized. These results were interpreted to support the view that a percept in the visual system develops gradually over time, permitting gross figure-ground differentiation very early in processing, but discrimination of fine detail only later. Implications of these results for human-factors applications are discussed.
\end{abstract}

If a subject is asked to discriminate among several different forms, how is the speed of the discrimination affected as the size of the forms is increased? Intuitively, we might expect that the speed of the discrimination would increase as the size increased within a limited range, but the basis for this intuition is not so readily apparent. We know that reaction time (RT) decreases as stimulus intensity increases from very weak to moderate levels, but in the present instance we are dealing with the discriminability between two stimuli. The discrimination between the letters " $A$ " and " $T$ " is based upon differences in their shapes (or their features) that seem to remain invariant as the size of the letters changes.

Difference in features becomes an acuity variable when the size of the features is manipulated. The visual acuity necessary to discern the details critical for discrimination of an " $A$ " from a " $T$ " decreases as the size of the target (and thereby, of the features) is increased.

The relation between visual acuity and discrimination time is apparent when temporal integration in the visual system is considered. The human visual system, like any biological system, lacks the capacity for infinitely fine temporal resolution. This fact provides the basis for Bloch's law of time-intensity reciprocity and has been reviewed at length by Ganz (1975). Put another way, maximum response in the visual system develops over an appreciable span of time. For simple flashes of light, the span is probably on the order of $100 \mathrm{msec}$ or less; but for form stimuli, Kahneman and his colleagues (Kahneman, 1964; Kahneman \& Norman, 1964;

The authors wish to acknowledge the assistance of Glenn Swimmer, who collected these data. This research was supported by Public Health Service Research Grant MH-1206 and by United States Public Health Service Research Career Program Award K6-MH-22014 to the second author. Requests for reprints should be addressed to Charles W. Eriksen, Psychology Building, University of Illinois, Champaign, Illinois 61820.
Kahneman, Norman, \& Kubovy, 1967) have shown that the span extends to $300 \mathrm{msec}$ or more. These findings imply that there is a gradual accumulation of information in the visual system. This conclusion is also supported by the speed-accuracy tradeoff reported in the visual processing and performance literature (Fitts, 1966; Pachella, 1974; Swensson, 1972): If a percept is gradually developing in the visual system, then the subject can terminate processing early for a fast response when relatively little information has accumulated or terminate processing later when a fully developed percept is available. Eriksen and Schultz (1978) have drawn the comparison of the gradually accumulative nature of a percept in the visual system to the development of a photographic print when bathed: Gross figure-ground differentiation occurs early in the process, and fine detail becomes apparent only much later, after sufficient energy has been accumulated.

It follows that the speed with which two forms can be discriminated from each other will depend upon the size of their differentiating features. If these features are quite small, a longer period of energy summation in the visual system will be needed to resolve the acuity requirement they present. Thus, increasing the size of the forms to be discriminated does not change the features that differentiate among them, but it should decrease the time required for a discrimination by reducing the energy-summation time required to discern the differentiating features.

\section{METHOD}

\section{Subjects}

Ten graduate and undergraduate students at the University of Illinois (seven male) served as paid volunteers. All had normal or corrected-to-normal vision and were right-handed. Several had prior experience with tachistoscopic displays.

\section{Apparatus and Stimuli}

Trials were conducted on a Scientific Prototype Model 800F 
two-channel card tachistoscope. Luminances in both channels were set at $32.1 \mathrm{~cd} / \mathrm{m}^{2}$, as measured by a Spectra spot photometer. Trials were initiated at the subject's discretion by his pressing a microswitch trigger held in the left hand. Trial initiation simultaneously onset the stimulus field and started a Hunter Model 120A Klockcounter set to time in milliseconds. Responses were recorded by the subject's pressing a toggle microswitch (located under his right hand) to the left or to the right. The stimulus remained on for $2 \mathrm{sec}$.

Twenty stimulus displays were constructed by placing one of four Paratype Futura demibold letters (A, V, H, or T) on blank white $5 \times 8$ in. file cards. The stimulus letters could appear in one of five sizes, subtending $.143, .214, .50,1.07$, or $2.14 \mathrm{deg}$ of visual angle (va) in height at the 32 -in. viewing distance. A fixation display was constructed by placing a faint pencil dot, $.03 \mathrm{deg}$ in diameter, centered where the target letters would appear. Half of the subjects were instructed to move the toggle switch to the right if the target letter was an $\mathrm{A}$ or a $\mathrm{V}$, and the remaining subjects were given the opposite response instruction.

\section{Procedure}

Each subject served in two sessions. Each session was conducted under either a blocked or a randomized condition of stimulus presentation. For the blocked condition, a block of $\mathbf{4 0}$ trials was run under one and only one letter size, with the four target letters randomized within a block. For the randomized condition, both letter size and targets were randomized within a block of 40 trials. A session consisted of five blocks of 40 trials each. Stimulus presentation (blocked or randomized) was counterbalanced across subjects, with block order counterbalanced within each level of stimulus presentation and across subjects. Each subject provided 40 trials for each cell of the experimental design, or 10 trials under each target letter.

Viewing was binocular, and trials were initiated at the subject's discretion following a "Ready" signal from the experimenter. Subjects were instructed to respond as quickly as possible, while avoiding errors, and were told that a $5 \%$ error rate was tolerable. Each session began with 20 practice trials on the largest letter size, and each block was preceded by 5 warm-up trials chosen from the last 5 trials in that block and run in reverse sequence. Latency feedback in milliseconds followed each trial, and a 5-min break followed the third block. Sessions typically lasted $55 \mathrm{~min}$.

\section{RESULTS AND DISCUSSION}

Mean target recognition latencies under both the blocked and the randomized stimulus presentation conditions are shown as a function of target size in Figure 1. These data were analyzed by a three-way mixed-model ANOVA (size by presentation condition by subjects). The main effect of target size was highly significant $[F(4,36)=19.14, p<.0001]$, al though the main effect of stimulus presentation only approached significance $[F(1,9)=4.55, p=.06]$. The interaction failed to approach significance.

For both blocked and randomized presentation conditions, recognition latency decreases as target letter size increases from .143 to approximately .75 deg va, at which point the functions appear to become asymptotic. These pooled results essentially characterize the performance of all 10 subjects: All subjects showed a decrease in recognition latency as target size increased, but they differed in the magnitude of latency facilitation, as well as in the point at which

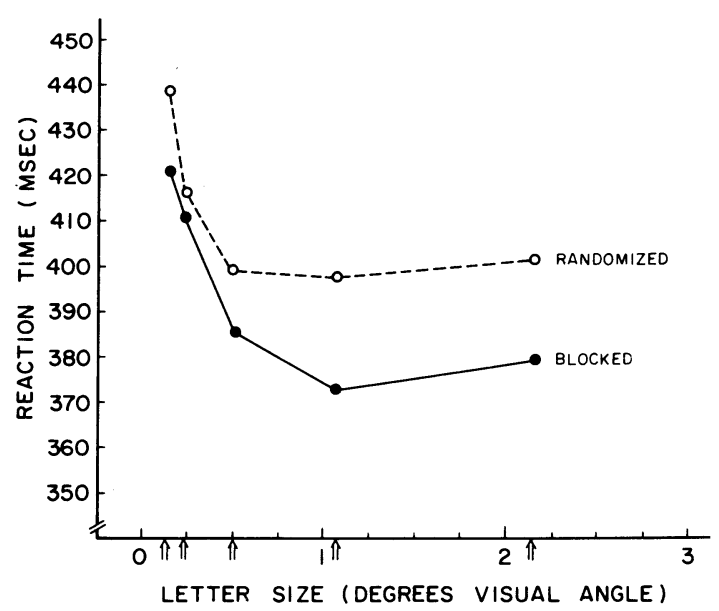

Figure 1. Mean latency to target recognition as a function of target size. The parameter is blocked vs. randomized presentation of stimulus sizes, and the data are pooled across the 10 subjects.

their function became asymptotic with target size.

Clearly, the effect of target size on recognition latency is robust. Further, this effect cannot be attributed to a speed-accuracy tradeoff: Mean percentage errors across the 10 experimental conditions are presented in Table 1. Speed and accuracy correlated at +.58 .

For both blocked and randomized presentation conditions, the latency reduction obtained by increasing target size is restricted to a limited size range. Apparently, when a target is big enough, there is no further gain in processing speed. Superficially, the functions are similar to those obtained with simple RT and stimulus intensity: Latency to a signal is reduced only over a limited range on the low-intensity end. However, in the case of choice RT, a different process may be responsible. As the targets increase in size, differentiating detail becomes larger, requiring less acuity to discern those critical details. But simultaneously, an opposing variable is operating: As the targets become larger, the critical details are moved further out into the fovea, where acuity decreases. Eriksen and Schultz (1977) have shown that recognition latency increases as target letters are presented at increasing distances from foveal center out to 3 deg va. To compensate for the loss in acuity, increased energy-summation time is required. Thus, the effect of target size may be viewed as a result of two opposing processes: (1) the decreasing acuity requirement that occurs as the differ-

Table 1

Percent Errors for Each Target Size Under Both Presentation Conditions

\begin{tabular}{lccccc}
\hline & \multicolumn{5}{c}{ Target Size (in Degrees of Visual Angle) } \\
\cline { 2 - 6 } & .143 & .214 & .50 & 1.07 & 2.14 \\
\hline Blocked & 7.75 & 5.50 & 6.75 & 6.25 & 6.50 \\
Randomized & 9.00 & 6.50 & 5.50 & 6.25 & 5.75 \\
\hline
\end{tabular}


entiating details of the forms becomes larger, and (2) the loss in acuity that occurs when those details fall further out on the fovea. However, a point is reached at approximately $.75 \mathrm{deg}$ va where increasing the size of the critical details no longer offsets the decrease in acuity of their placement on the retina: There is a range where RT remains constant as target size increases. $\mathrm{Had}$ the experiment varied size over a wider range, one might have expected that latency would have again increased. In fact, there is a suggestion of this in these data, although the increase in latency at the largest target size is not significant.

Alternatively, when the subject knows that the target letters will all be the same size in a block of trials, recognition latency is probably shorter than if all sizes are intermixed in a trial block. The difference between blocked and randomized presentation is least for the smallest targets and increases to a constant value as target size approaches $.75 \mathrm{deg}$ va.

Although the main effect of blocked vs. randomized stimulus presentation only approached significance, there are several reasons why we believe it to be a real effect. First, 8 of the 10 subjects were faster under blocked than under randomized presentation, although the magnitude of the latency reduction from blocking trials differed among them. The two subjects who showed the opposite effect reported no prior tachistoscopic experience. Second, the superior performance obtained with blocked presentation was not unanticipated. Eriksen and Eriksen (1974) obtained faster RTs when target letters and noise background were blocked in terms of type of noise. In the present experiment, we feel the effect may reflect an attentional function. Eriksen and Hoffman (1974) have shown that the recognition latency for a single target letter is shorter if the subject knows the precise location in the field where the letter will appear. Notably, this effect is independent of eye fixation. Somewhat similar results have been reported by Posner, Nissen, and Ogden (Note 1) for detection tasks. In the present experiment, the subject knew that the letters would be of a constant size for all the trials in a block. It may be that he could attend to the part of the visual field where the differentiating features of the letters were more apt to appear. Indeed, the magnitude of the difference between blocked and randomized presentation at the point where the functions become asymptotic is $23 \mathrm{msec}$, which is comparable to the $28 \mathrm{msec}$ obtained by Eriksen and Hoffman (1974) for the difference between conditions when (1) the subject knew the location of the target before the trial, as opposed to (2) the location of the target was randomized.

Third, we argue that the main effect of stimulus presentation is real because the asymptotic level of recognition latency for blocked trials is significantly faster than that for randomized presentation of targets. Since the exact point of asymptote for the two functions shown in Figure 1 is uncertain, the mean latencies of target recognition were pooled over the two largest target sizes (1.25 and $2.25 \mathrm{deg})$ for each function. The resultant mean asymptotic latencies for blocked and randomized presentations were 377 and $399 \mathrm{msec}$, respectively. A planned comparison of this difference was significant $(p<.05)$. Since $(1)$ the asymptotic levels of blocked vs. randomized presentation differed significantly, (2) this effect of stimulus presentation is consistent with previous results, and (3) all the experienced subjects performed faster with blocked trials, we argue that the main effect of stimulus presentation, though marginal, is real.

These data also bear implications for human-factors applications, especially in display design. First, increasing the size of a display character beyond 1.0 deg va will not render it more quickly processed. Second, characters should be of relatively constant size throughout a display.

\section{REFERENCE NOTE}

1. Posner, M. I., Nissen, M. J., \& Ogden, W. C. Attended and unattended processing modes: The role of set for spatial location. Paper presented at SSRC Conference on Perceptual Modes, Minneapolis, Minnesota, June 1975.

\section{REFERENCES}

Eriksen, B. A., \& Eriksen, C. W. Effects of noise letters upon the identification of a target letter in a nonsearch task. Perception \& Psychophysics, 1974, 16, 143-149.

Eriksen, C. W., \& Hoffman, J. E. Selective attention: Noise suppression or signal enhancement? Bulletin of the Psychonomic Society, 1974, 4, 587-589.

Eriksen, C. W., \& Schultz, D. W. Retinal locus and acuity in visual information processing. Bulletin of the Psychonomic Society, 1977, 9, 81-84.

Eriksen, C. W., \& Schultz, D. W. Temporal factors in visual information processing. In J. Requin (Ed.), Attention and performance VII. New York: Academic Press, 1978.

FrrTs, P. M. Cognitive aspects of information processing: III. Set for speed versus accuracy. Journal of Experimental Psychology, 1966, 71, 849-857.

GANZ, L. Temporal factors in visual perception. In E. C. Carterette \& M. P. Friedman (Eds.), Handbook of perception (Vol. 1). New York: Academic Press, 1975.

Kahneman, D. Temporal summation in an acuity task at different energy levels-A study of the determinants of summation. Vision Research, 1964, 4, 557-566.

Kafneman, D., \& Norman, J. The time-intensity relation in visual perception as a function of the observer's task. Journal of Experimental Psychology, 1964, 68, 215-220.

Kamneman, D., Norman, J., \& Kubovy, M. The critical duration for the resolution of form: Centrally or peripherally determined? Journal of Experimental Psychology, 1967, 73, 323-327.

Pachella, R. The interpretation of reaction time in information-processing research. In B. Kantowitz (Ed.), Human information processing: Tutorials in performance and cognition. Hillsdale, N.J: Erlbaum, 1974.

Swensson, R. G. The elusive tradeoff: Speed versus accuracy in visual discrimination tasks. Perception \& Psychophysics, 1972, 12, 16-32.

(Received for publication August 16, 1978.) 